\title{
RECHERCHES SUR QUELQUES TRÉMATODES MONOGÉNÈSES NOUVEAUX OU PEU CONNUS
}

\author{
Par Louis GALLIEN
}

\section{Introduction}

Les trématodes monogénèses étudiés dans ce travail ont été récoltés par moi-même en juin 1934, au cours de la deuxième croisière océanographique du "Président Théodore-Tissier». Celle-ci s'est déroulée dans la mer du Nord et l'Atlantique Est, autour des Iles Britanniques : de Copenhague à Brest, par les Orcades, Stornoway (Ile Lewis), Belfast, Glengariff (côte Ouest de l'Irlande).

Les poissons qui hébergeaient les parasites, ont été examinés dès leur capture. Les vers ont été cbservés d'abord sur le vivant. Quand la récolte comportait plusieurs individus, un premier lot a été fixé en extension et monté in toto après coloration par l'hémalun dilué, un second lot, fixé au Bouin, a été débité en coupes sériées d'une épaisseur de $5 \mu$ et $7 \mu$ suivant les cas. Il a été fait des coupes transversales, sagittales et frontales, toutes les fois qu'il a été possible. Ceci est presque indispensable pour comprendre l'anatomie de l'appareil génital. Les coupes ont été colorées au glychémalun-éosinevert lumière.

L'examen des différentes espèces recueillies et la revue bibliographique que j'ai faite m'ont amené, en dehors de la description de formes nouvelles, à réétudier une espèce décrite autrefois d'une manière insuffisante et à revoir la systématique de quelques familles. Au cours de la croisière, j'ai recueilli un certain nombre d'espèces connues, dont il n'y avait pas lieu de parler ici, sinon d'une manière incidente.

Je remercie M. Le Danois, Directeur de l'Office des Pêches, ainsi que le Commandant Beaugé, pour l'accueil très courtois reçu à bord du « Th.-Tissier ». Leurs collaborateurs ont bien voulu s'associer à leur aimable réception. Ils ont, de plus, vérifié la détermination des poissons examinés.

AnNales de Parasitologie, T. XV, $\mathrm{N}^{0} 1 .-1^{\mathrm{er}}$ janvier 1937 , p. 9-28. 


\section{FAMILLE DES MONOCOTYLIDE TASCH}

\section{Sous-Famille des « Pseudocotylina 》 Monticelli}

\section{Pseudobothrium pristiuri n. g. n. sp.}

Récolte. - Parasite de Pristiurus melanostomus Raf.

Station 175 : $53^{\circ} 17,13^{\circ} 08$. Au chalut par $260 \mathrm{~m}$. de fond. Le 24-6-34. Fond à Spatangus purpureus. Station $170: 55^{\circ} 55,7^{\circ} 42$. Au chalut par $138 \mathrm{~m}$. de fond. Le 21-6-34. Fond à Dentalium et Hyalinœcia. Ces stations se trouvent au large et à l'ouest du Sud de l'Irlande.

\section{Morphologie externe (Pl. I, fig. 3) (1)}

Les vers sont fixés sur la peau, dans la région du dos, et ont l'aspect de petites écailles brunâtres. Ils sont assez faciles à détacher.

La longueur du corps est légèrement variable, les plus grands animaux ont $1 \mathrm{~mm} .6$ de long et $1 \mathrm{~mm}$. de large, mais les rapports entre les dimensions du corps sont modifiables, par suite des contractions de l'animal.

La forme du corps est celle d'une feuille lancéolée, les deux extrémités étant acuminées. Dans la région antérieure et ventralement, s'ouvre la bouche $(B$.$) , qui présente deux lèvres circulaires, mais$ ces bourrelets utilisés dans la fixation n'ont cependant pas la structure d'une ventouse. Dans sa partie postérieure et ventralement, le corps porte une petite ventouse $(V$.$) circulaire, inerme,$ qui fait à peine saillie sur les téguments. Elle mesure $100 \mu$ de diamètre, elle est donc très discrète.

C'est par la bouche et la petite ventouse que se tient l'animal, mais il est surtout étroitement appliqué sur les téguments de l'hôte par toute sa surface ventrale, unie, et régulière.

Morphologie interne (Fig. 1 et Pl. II, fig. 8 et 9)

Appareil digestif. - La bouche (B.) donne accès dans un pharynx $(P h$.$) très développé, et qui présente une structure particu-$ lière. Dans l'ensemble, il a l'aspect d'un cône allongé. Une première partie est constituée par une masse musculaire, au sein de laquelle la lumière digestive a l'aspect, en section transversale, d'une étoile à trois branches. Puis le diamètre du pharynx s'élargit et la masse musculaire se creuse de vastes cavités obliques, de

(1) Les planches paraîtront avec la deuxième partie de ce mémoire, dans le $\mathrm{n}^{\circ} 2$ de ces Annales (mars 1937). 
telle sorte que l'ensemble prend un aspect lacuneux. Ce caractère est bien spécial à l'espèce décrite ici. Je ne l'ai pas retrouvé chez les autres genres de la famille que j'ai étudiés (Microbothrium, Leptocotyle).

Le pharynx s'ouvre dans un œsophage, qui se divise immédiatement en deux branches latérales $(T . d$.), enserrant l'appareil génital, pour se rapprocher vers l'arrière sans cependant fusionner. Chaque branche émet sur sa face externe une série de diverticules profonds, à peine découpés, qui forment huit à neuf digitations. Ces digitations apparaissent, sur les préparations in toto, en clair sur le fond sombre de la glande vitellogène. La face interne des anses intestinales n'émet aucune digitation.

L'épithélium qui tapisse l'intestin est constitué par des cellules polyédriques nucléées, à contenu clair, qui se détachent successivement pour tomber dans la lumière digestive.

Appareil excréteur. - Dans la région céphalique de l'animal, sur les côtés et au niveau du pharynx, on trouve deux vésicules excrétrices (V.e.) assez vastes, qui s'ouvrent dorsalement par un pertuis étroit. Je n'ai pu suivre à l'intérieur du corps le prolongement de ces vésicules excrétrices.

Système nerveux. - La bande-

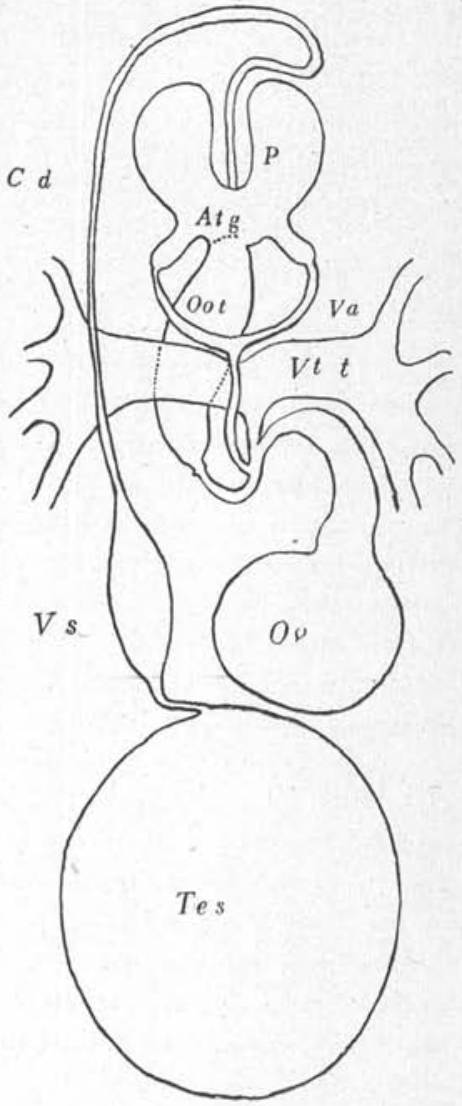

Fig. 1. - Appareil génital de Poetdobolhrium pristiuri. L'ootype, normalement médian, est rejeté sur le côté pour montrer le canal vaginal. Vue ventrale. lette cérébrale est bien développée (pl. I, fig. 3 et pl. II, fig. 8 c.). Elle est située dorsalement, au-dessus du pharynx antérieur. Elle se prolonge par deux trones céphaliques. Vers l'arrière, elle donne une paire nerveuse qui longe le pharynx, les anses intestinales et qui émet latéralement plusieurs ramifications. Les deux branches deviennent coalescentes en avant de la ventouse. 
Téguments, Parenchyme. - Un caractère très net de cet ectoparasite, c'est la grande épaisseur de la cuticule (pl. II, fig. 8 et 9). Celle-ci forme une couche anhiste, épaisse de $40 \mu$ sur le dos, de $25 \mu$ sur le ventre. Au niveau de la ventouse, elle se différencie en une membrane plus rigide.

Le parenchyme n'est bien représenté que dans la région céphalique et postérieure. Il forme par ailleurs des trabécules dorso-ventraux qui enserrent l'appareil génital. Quelques fibres musculaires longitudinales se voient au voisinage de la ventouse.

Appareil génital (fig. 1 et pl. II, fig. 8 et 9 ) :

1) Appareil mâle. - Le testicule (Tes.) est unique, constitué par un gros cyste sphérique qui occupe toute l'épaisseur du corps et a un diamètre de $140 \mu$. Il est situé en arrière, dans le cul-de-sac limité par les deux anses intestinales. Il en part un court canal qui, très vite, se renfle en une vésicule séminale $(V . s$.) remplie de sperme. Cetțe vésicule se poursuit par un canal déférent $(C . d$.) qui se dirige vers l'avant. Dans la région antérieure, un peu avant d'atteindre l'œsophage, le canal déférent décrit une courbe qui le ramène vers l'arrière, où il se prolonge dans un gros pénis $(\boldsymbol{P}$.). Celui-ci pend dans un atrium génital (At. g.), où viennent s'ouvrir également les vagins et l'utérus.

2) Appareil femelle:

a) Ovaire et voies génitales femelles. - L'ovaire $(\mathrm{Ov}$.) est petit, situé juste devant le testicule, et un peu sur le côté. Il se prolonge par un court oviducte jusqu'à un point de confluence, où s'ouvre le canal vaginal et le vitelloducte. De ce point, part un canal qui se retourne vers l'avant et mène dans une vaste poche médiane, dont il est séparé par un sphincter. Cette poche (Oot.), tapissée de glandes, qui, de dorsale et antéro-postérieure qu'elle était, devient brusquement dorso-ventrale au moment où elle s'ouvre dans l'atrium génital, doit être considérée comme un vaste ootype.

b) Annexes de l'appareil femelle. - Deux vagins (Va.) s'ouvrent latéro-ventralement dans l'atrium génital. Ils se dirigent en arc vers l'arrière, pour se réunir sur la médiane et donner naissance à un canal vaginal simple très ténu. Ce canal se dirige vers l'arrière et, après s'être un peu élargi, s'ouvre au point de confluence de l'oviducte.

La glande vitellogène $(V t$.) est très développée. Elle occupe tout l'espace compris entre les diverticules intestinaux. Les lobes vitellins confluent vers deux grosses branches transverses (Vt. $t$.), 
situées entre l'atrium génital et l'ovaire. Ces deux branches confluent et s'ouvrent sur la médiane au point commun, où viennent déboucher également l'oviducte et le canal vaginal.

\section{Revue Systématioue}

La famille des Monocotylidæ Tasch. est actuellement (Fuhrmann 1929, Sprehn 1933) divisée en deux sous-familles :

a) Pseudocotylinæ Monticelli 1903 : pas de ventouses à l'extrémité antérieure du ver, pas de septes à la ventouse postérieure.

b) Monocotylinæ Monticelli (1903) : ventouse postérieure avec septes. Nous laisserons de côté cette sous-famille.

Dans les Pseudocotylinæ, il a été décrit les genres suivants :

Pseudocotyle van Ben. Hesse (1863, 4" Appendice) avec Ps. squatinæ, pour un trématode parasitant la peau du dos de Squatina angelus. Ce genre est caractérisé par l'allongement du corps et la présence de nombreux testicules.

Microbothrium Olsson (1870) avec M. apiculatum, pour un parasite de la peau du dos d'Acanthias vulgaris.

Microbothrium est allongé et ne possède qu'un gros testicule. Taschenberg (1878) pense que ces deux genres sont identiques, mais Saint-Rémy (1891) a réétudié l'appareil génital de l'espèce décrite par Olsson. Il conclut à la réalité du genre. J'ai recueilli deux individus de $M$. apiculatum au cours de la croisière. Leur étude me permet de confirmer les conclusions de Saint-Rémy et la validité de l'espèce d'Olsson. En particulier, il n'existe qu'un testicule.

Lintonia Monticelli (1904 b) avec L. papillosa Linton (1898) pour un parasite de Gadus callarias, décrit primitivement comme Nitzschia papillosa par Linton en 1898.

Anoplodiscus Sonsino (1890) avec $A$. richiardii, parasite de Pagrus orphus. Cette espèce a été réétudiée par Monticelli $(1904$ a $)$.

Ces deux derniers genres ont un testicule, mais semblent aberrants par la présence de fossettes glandulaires dans la région antérieure du corps, qui, à considérer les figures, ressemblent à des ventouses.

Leptocotyle Monticelli (1890) avec $L$. minor, pour un parasite de Scyllium canicula. Cette espèce est caractérisée par un corps court, un seul testicule, une poche du cirre complexe.

Ce genre et cette espèce ont été l'objet de multiples erreurs. J'en 
ai récolté, pendant la croisière, de nombreux échantillons, dont l'étude m'a permis d'éclaircir une synonymie confuse.

Monticelli, en 1890, décrit tout d'abord l'espèce sous le nom de Pseudocotyle minor, ce qui est une première faute puisqu'il n'existe qu'un testicule et que Pseudocotyle van Ben. Hesse en a plusieurs. En 1904 (a), Monticelli, dans une note infra-paginale, scinde son genre Pseudocotyle en trois :

Pseudocotyle s.s. avec Ps. squatinæ Van Ben. Hesse.

Microbothrium Olsson avec $M$. apiculatum.

Leptocotyle. Ce genre est destiné implicitement, car Monticelli ne reconnaît pas formellemnt son erreur, à son Pseudocotyle minor, qui doit devenir Leptocotyle minor Monticelli (1890).

Enfin Johnstone (1911) réétudie un parasite décrit par Scott (1906) comme Epibdella et il décrit Paracotyle avec $P$. caniculæ, parasite de la peau de Scyllium canicula. La comparaison que j'ai pu faire montre à l'évidence qu'il s'agit de Leptocotyle minor, mais Johnstone n'a pas eu connaissance des travaux de Monticelli.

Jones (1933) a fort bien repris l'étude anatomique de cette espèce, mais il ignore aussi les travaux de Monticelli. Il pense que le Paracotyle de Johnstone est le Microbothrium d'Olsson et il donne à cette espèce le nom de $M$. caniculæ, ce qui est une nouvelle erreur commise déjà dans une communication préliminaire faite avec Baylis (1933).

En somme, le parasite de Scyllium canicula doit s'appeler Leptocotyle minor Monticelli (1890). Les autres ncms sont erronés et Paracotyle doit disparaître.

Le genre Pseudobothrium décrit ici avec Pseudobothrium pristiuri est nettement caractérisé :

$1^{\circ}$ par la forme et la taille du corps, ce qui l'éloigne de Pseudocotyle et Microbothrium;

$2^{\circ}$ par l'existence d'un seul testicule, ce qui le différencie de Pseudocotyle ;

$3^{\circ}$ par l'existence de deux vagins et d'un cirre simple, ce qui le différencie de Leptocotyle et Microbothrium ;

$4^{\circ}$ par l'existence de ramifications intestinales, caractère opposé à celui de Leptocotyle. 


\section{FAMILLE DES “ DICLIDOPHORIDA 》 CERF.}

Diclidophoropsis tissieri n. g. n. sp.

Récolte. - Parasite de Macrurus lœvis Lowe.

Station 175 (voir la référence ci-dessus à Pseudobothrium). Le chalut ramène dans cette région d'assez nombreux individus de Macrurus loevis Lowe, 13 Macrurus ont été examinés. La plupart sont parasités par cinq ou six individus de Diclidophoropsis. Une vingtaine d'exemplaires de taille diverse ont été fixés.

\section{Morphologie externe (Pl. I, fig. 2)}

Les vers sont fixés sur la peau dans la région des branchies, mais non sur celles-ci. Ils sont facilement visibles. La longueur du corps atteint 6 à $7 \mathrm{~mm}$. lorsqu'ils sont en extension moyenne, la largeur est de $1 \mathrm{~mm}$. Il existe sur le mème poisson, à côté d'individus adultes, des individus jeunes dont la longueur est de $3 \mathrm{~mm}$. 5, la largeur $0 \mathrm{~mm}$. 7 .

Le corps présente une symétrie bilatérale, il est allongé, plat, d'un blanc jaunâtre sur les bords, plus hyalin dans la région centrale. Il s’atténue progressivement dans la région céphalique.

Dans la région antérieure, on observe d'abord deux ventouses ovalaires ventrales ( $V$.$) entourant la bcuche (B$.$) ventrale éga-$ lement. Plus en arrière, légèrement après la poche du cirre (Ci.), on observe sur les bords latéraux du ver une légère constriction, au fond de laquelle on trouve de chaque côté une sorte de plissement des téguments. Ce plissement marque l'entrée des deux vagins (Va.).

Dans sa partie postérieure, le corps se continue en un plateau fixateur. Ce plateau porte normalement huit ventouses formant quatre paires pédiculées, disposées radiairement. Chaque pédicule est long, extensible et très déformable. Dans de nombreux cas, surtout chez les grands individus, une ou deux ventouses manquent et ne sont plus représentées que par une portion plus ou moins longue de leur pédicule. L’extrémité distale de celui-ci, dans ce cas, est normalement cicatrisée, cette disposition étant vraisemblablement le résultat d'un accident.

Structure des organes adhésifs:

Ventouses orales. - Les ventouses antérieures communiquent largement avec la cavité buccale. Elles sont ovales, les deux axes 
mesurant respectivement $250 \mu$ et $175 \mu$. Chaque ventouse est constituée par une cupule assez épaisse, dont les fibres museulaires sont perpendiculaires à la surface.

Ventouses postérieures (fig. 2). - Celles-ci sont circulaires, parfois légèrement étirées suivant l'axe principal de leur pédoncule. Elles ont un diamètre de $750 \mu$. Leur structure est complexe et tout à fait caractéristique de celle qui est propre à la famille des Diclidophoridx.

Chaque organe est pourvu d'une charpente rigide qui le main-

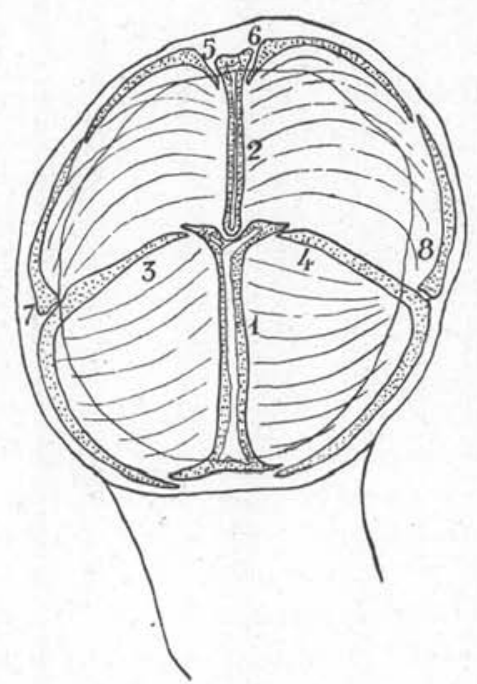

Fig. 2. - Ventouse de Diclidophoropsis tissieri, la cupule musculaire n'est pas représentée.

tient cuvert: il fonctionne comme ventouse uniquement et non pas comme "ventouse-pince », ainsi que cela se passe dans d'autres formes (Octocotylidx).

Il existe huit pièces de soutien, qui donnent à l'ensemble de la charpente l'aspect d'une croix entourée d'un cercle.

Suivant l'axe longitudinal de la ventouse, on trouve d'abord deux pièces légèrement arquées, la proximale (1) étant plus longue et plus épaisse que la distale (2) et se terminant à ses deux extrémités par une fourche très ouverte. Ces deux pièces sont creuses.

Du centre de la ventouse, et s'appuyant sur l'extrémité distale de la pièce 1, partent deux pièces (3 et 4 ), qui se dirigent vers le 
bord de la ventouse. A cet endroit, chaque pièce s'infléchit suivant un angle voisin de $90^{\circ}$ et se prolonge par une branche qui suit le bord de la ventouse, pour venir toucher par sa pointe l'extrémité proximale de la pièce 1 .

De chaque côté de l'extrémité distale de la pièce 2 part une pièce en are (5 et 6 ) qui longe le bord de la ventouse, suivant un arc de $45^{\circ}$ environ.

Enfin, du point d'inflexion de chacune des pièces 3 et 4, part une pièce en arc ( 7 et 8 ) qui longe le bord de la ventouse et va rejoindre l'extrémité acuminée des pièces 5 et 6 .

Les huit pièces sont grêles, contrairement à ce qu'on observe chez plusieurs autres genres de la famille. La pièce 1, chez d'autres espèces, dessine une courbe beaucoup plus accentuée. En somme, chaque ventouse a la forme d'une cupule aplatie et largement ouverte.

Les pièces de soutien étayent une cupule musculaire qui tapisse tout le fond de la ventouse. Les fibres de cette cupule sont normales à la surface. Je n'ai pas observé de stries dans ces fibres. Cerfontaine a décrit des fibres striées chez Diclidophora labracis Cerf. (1895) et dans la ventouse postérieure d'un tristomide : Merizocotyle diaphanum Cerf. (1894). Sur cette cupule, s'insèrent de longues et épaisses bandes musculaires longitudinales et obliques qui traversent le pédoncule de chaque ventouse et vont se perdre dans le parenchyme de la région postérieure du corps.

Morphologie interne (Pl. I, fig. 2, Pl. II, fig. 5, 6, 7)

Appareil digestif. -- La bouche $(B$.) donne accès dans un entonnoir buccal, qui mène à un pharynx $(P h$.), épais, et très développé. Dans la partie postérieure de celui-ci, des cellules glandulaires sont différenciées (fig. 6, pl. II, Gl. Ph.).

Le pharynx s'ouvre dans un œsophage qui s'étend sous le pharynx et se bifurque très vite pour donner deux branches intestinales latérales. Celles-ci confluent à nouveau dans la région du plateau adhésif. Des branches principales partent de nombreuses digitations. On en trouve dans le pédoncule de chaque ventouse. Le tube digestif est tapissé par un épithélium de cellules claires. Dans les animaux coupés, le tube digestif était vide.

Appareil excréteur. - Il est constitué par deux canaux latéraux, qui s'ouvrent sensiblement au niveau des vagins et dorsalement, par un orifice étroit. Celui-ci mène dans une vésicule $(V . e$.$) plus$ 
large, allongée, appliquée contre le tube digestif au point où celuici se bifurque (pl. II, fig. 7 C.e).

La vésicule se continue postérieurement par un canal situé ventralement, sous chaque branche du tube digestif, canal qui se prolonge jusqu'à la région du plateau adhésif, où j'ai cessé de le suivre.

Système nerveux. - La bandelette cérébrale $(C$.) se trouve située dorsalement, au-dessus de la moitié antérieure du pharynx. Elle est bien développée. Il en part̂̀ deux troncs antérieurs et quatre postérieurs et ventraux. Ceux-ci constituent deux paires : la première, que nous appellerons externe $(n . e$.$) , est tout à fait latérale.$ Chaque nerf est grêle et se termine dans le tiers postérieur du corps. La seconde paire, interne celle-là $(n . i$.), est beaucoup plus développée (pl. II, fig. 7). Chacun des nerfs latéraux qui la composent, est situé sous la branche correspondante de l'intestin. Les deux nerfs se rejoignent dans la région du plateau adhésif et envoient à ce niveau un filet nerveux dans le pédoncule de chaque ventouse.

Muscles et Parenchyme. - Une tunique musculaire (pl. II, fig. 7, M) enveloppe l'animal. On distingue sous une mince cuticule une couche très légère de fibres longitudinales, puis une couche de fibres circulaires et obliques, plus développée, et enfin une couche épaisse de fibres longitudinales. Celles-ci, surtout dans la région céphalique, s'isolent parfois en champs différenciés.

La tunique musculaire ventrale est plus développée que la dorsale. Il a été indiqué ci-dessus, à propos des ventouses, le grand développement musculaire qu'on trouve dans les pédicules des organes adhésifs.

Il convient enfin de signaler un caractère particulier à la région ventrale. Dans toute-la longueur du ver, depuis l'œsophage jusqu'au plateau adhésif, on trouve dans la région médio-ventrale un épaississement important de parenchyme, qui enrobe d'épaisses fibres musculaires longitudinales.

Le parenchyme est fort peu développé dans la plus grande partie du corps. Il n'est bien représenté que dans la région céphalique et dans celle du plateau adhésif.

Appareil génital (pl. I, fig. 2 ; pl. II, fig. 5, 6, 7 et fig. 3 du texte)

A ppareil mâle. - Les cystes testiculaires (Tes.) sont nombreux. Ils sont répartis dans la région postérieure du corps, entre les branches de l'intestin, depuis le niveau de l'appareil génital femelle, jus- 
qu'au point de confluence des branches latérales de l'intestin. Ils occupent toute l'épaisseur du corps.

Dans la partie supérieure de l'épaississement musculo-paren-

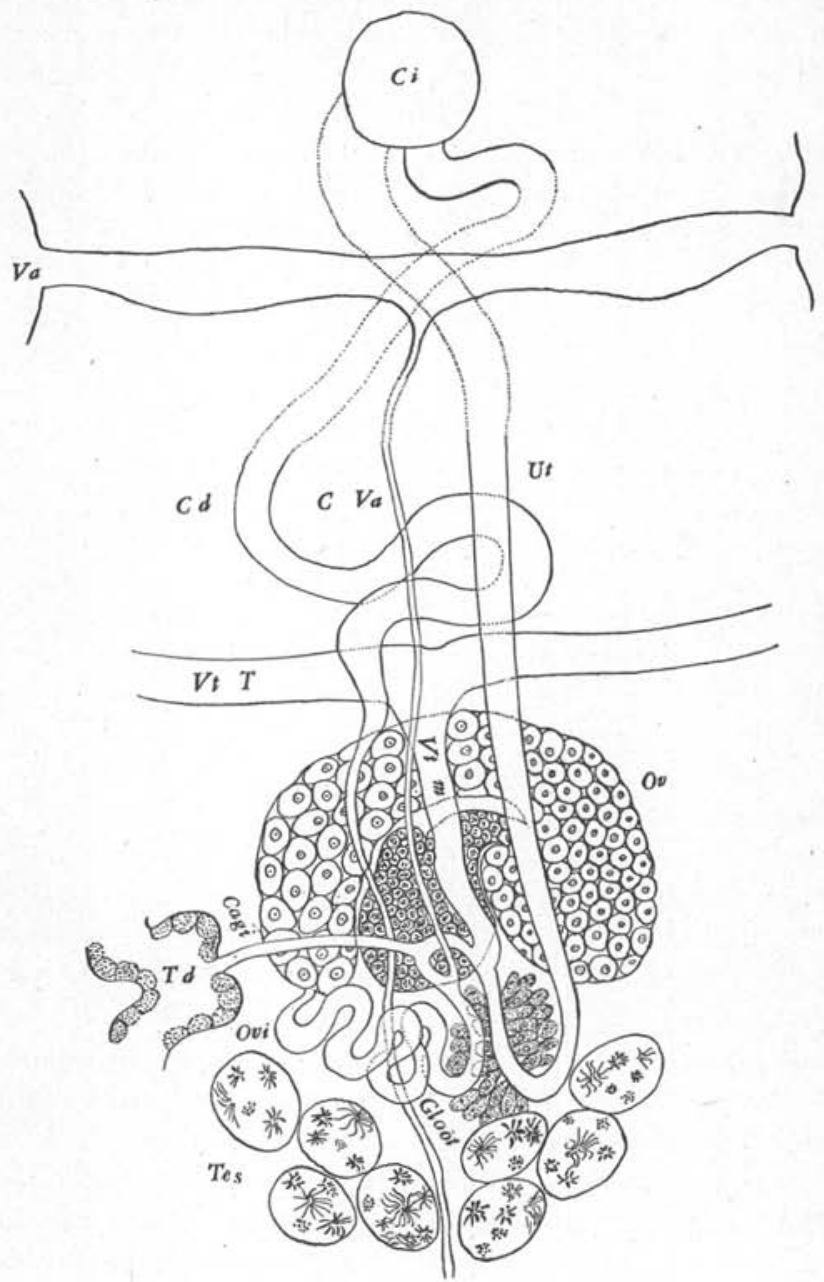

Fıc. 3. - Appareil génital de Diclidophoropsis tissieri. L'utérus,

le canal vaginal et le canal déférent sont très raccourcis (v. pl. I, fig. 2).

chymateux signalé ci-dessus, on trouve un canal très mince qui se précise de plus en plus, lorsqu'on gagne la région antérieure du corps. Il s'agit du canal déférent $(c . d$.$) , qui passe sous l'ovaire,$ s'élargit considérablement à cet endroit et se poursuit ensuite en un 
canal très sinueux rempli de sperme, parfaitement visible, pour déboucher ventralement dans la poche du cirre.

Le cirre (fig. 4) est une poche musculeuse, épaisse, située en arrière du pharynx, et qui s'ouvre à l'extérieur par un canal étroit.

Un caractère essentiel du cirre, qui caractérise le nouveau genre, est constitué par sa garniture de crochets. Il existe 128 crochets simples, disposés en couronne autour de la poche du cirre. Le nombre de 128 doit être entendu avec une approximation de 5 en plus ou en moins. Les crochets sont en effet extrêmement serrés et dif-
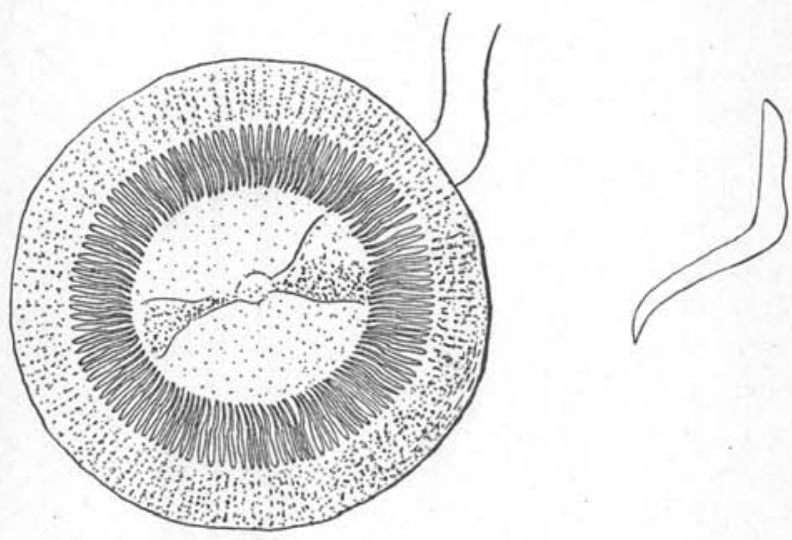

Fig. 4. - Le cirre et un erochet du cirre de Diclidophoropsis tissieri.

ficiles à compter. Ils ont l'aspect d'une lame coudée à angle obtus et terminée en griffe. La longueur totale de chaque crochet est de $25 \mu$.

Appareil femelle: 1) L'ovaire et les voies génitales femelles. L'ovaire $\left(O_{v}\right.$. $)$ est situé légèrement en avant du milieu du corps. Il comprend trois régions: d'abord un lobe médian qui contient de très jeunes ovocytes. Ce lobe se rétrécit dans sa région antérieure et mène dans un premier lobe latéral plus développé, où les ovocytes sont plus gros. Ce lobe se réfléchit antérieurement de façon à coiffer le lobe médian, pour venir former de l'autre côté du corps un second lobe latéral. Dans celui-ci, on trouve des ovocytes ayant acquis leur taille définitive.

De ce dernier lobe, part un canal contourné, l'oviducte, d'abord étroit, puis s'élargissant en se contournant davantage, pour devenir à nouveau relativement étroit au moment où il va recevoir d'autres canaux avant de s'ouvrir dans l'ootype. La région renflée et très 
contournée de ce canal contient des spermatozoïdes, et doit être interprétée comme un réceptacle séminal.

L'ootype (oot.) est situé dorso-ventralement (pl. II, fig. 7). Il est entouré de glandes annexes (Gl. oot.), et s'ouvre par un pertuis qui va en s'élargissant dans l'utérus. L'utérus (Ut.) est un canal long et large, situé ventralement et qui va s'ouvrir sous la poche du cirre. J'y ai trouvé des débris de coquille, et dans un cas un œuf muni de courts prolongements polaires et qui n'est peut-être pas très typique.

2) Annexes de l'appareil femelle: Vagins et canal vaginal. Un caractère essentiel de la forme étudiée ici, est l'existence d'un double vagin, et d'un canal vaginal inconnus jusqu'ici chez les autres genres et espèces de la famille.

Dans la région céphalique, légèrement en arrière du cirre et ventralement, on observe de chaque côté du corps un orifice qui mène dans un canal transversal assez large (pl. II, fig. 5 et 6). Les deux vagins $(V a$.), issus de chacun des orifices vaginaux, s'élargissent et se réunissent dans la région médiane, dorsalement, en une poche dans laquelle j'ai trouvé à plusieurs reprises des paquets de sperme (pl. II, fig. $5, S p$. ), ainsi que dans les vagins d'ailleurs. De cette poche, part vers l'arrière un canal très étroit, qui plonge d'abord jusque vers la région ventrale, et se continue postérieurement parmi les méandres du canal déférent. Il tranche sur ceux-ci par sa taille, et par son tracé un peu sinueux (pl. II, fig. 5 c. va.). Ce canal vaginal se poursuit sous l'ovaire, pour aboutir au réceptacle séminal, où il amène le sperme des vagins.

Appareil vitellin. - Il est constitué par une glande bien développée $(V t$.) située sur chacun des côtés du corps, autour des anses intestinales. Elle s'étend de la région des vagins jusqu'au plateau adhésif. Un peu en avant de l'ovaire et dorsalement, on observe un vitelloducte transverse $(V t . t$.), qui collecte les viteilocytes provenant des deux parties de la glande. Les deux branches transverses se réunissent en une branche médiane on viteiloducte médian ( $V t$. m.), qui passe sous l'ovaire et vient débcucher ventralement dans l'ootype.

Canal génito-intestinal. - C'est un canal étroit, qui, partant de la région où l'oviducte reçoit le vitelloducte médian, diverge brusquement (ca. g. i.) pour déboucher latéralement dans une anse intestinale ( $T$. d.). 


\section{Diclidophora pagelli $\mathrm{n}$. sp.}

Récolte. - Parasite de Pagellus centrodontus Delaroche.

Station 175 (voir ci-dessus pour Pseudobothrium et Diclidophoropsis). Un Pagellus examiné héberge un Diclidophora pagelli. Le seul exemplaire recueilli a été examiné in toto après traitement par le salicylate de méthyle, puis débité en coupes transversales.

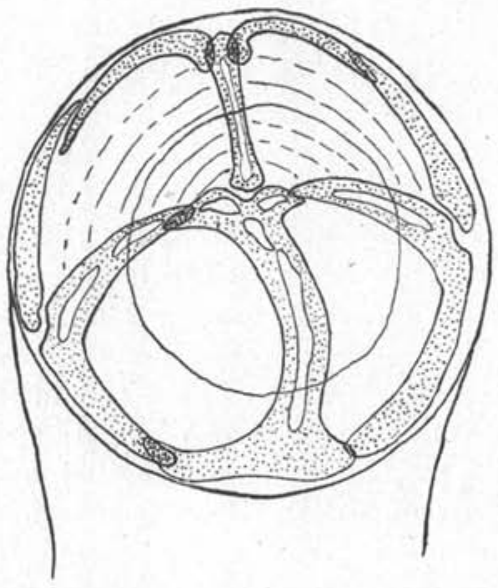

Fig. 5. - Ventouse de Diclidophora pagelli.

Morphologie externe (Pl. I, fig. 4)

L'animal est fixé sur les branchies de son hòte. La coloration générale du corps est brun noir. La longueur totale est de $3 \mathrm{~mm} ., 4$, la plus grande largeur de $1 \mathrm{~mm}$., 1 . Le corps est épais, largement arrondi dans la région antérieure, légèrement rétréci postérieurement. Le plateau adhésif est allongé et mesure $0 \mathrm{~mm}$., 9 ; il est plus large à sa base qu'à son extrémité. Il porte huit ventouses disposées en quatre paires. Les ventouses sont portées à l'extrémité de pédoncules épais, arrondis. Les pédoncules antérieurs sont plus longs que les postérieurs.

Dans la région antérieure, on voit la bouche ventrale (B.), à laquelle fait suite une paire de ventouses arrondies (V.). On n'observe pas d'orifices vaginaux.

Structure des organes adhésifs. - Elle est très comparable à celle de l'espèce décrite ci-dessus et des autres formes de la famille d'ailleurs. 
Ventouses orales. - Elles communiquent avec la cavité buccale, elles sont arrondies et sont constituées par une cupule musculaire béante.

Ventouses postérieures. - Elles sont circulaires, épaisses, leur concavité est fortement accentuée. Elles ont un diamètre de $350 \mu$ environ.

Chaque organe est pourvu d'une charpente rigide qui assure la tenue de l'ensemble. Cette charpente est exactement comparable à celle qui a été décrite pour Diclidophoropsis ainsi que le montre la fig. 5. Il n'y a donc pas lieu de reprendre cette description. II convient de signaler toutefois que les pièces sont plus épaisses et que la pièce axiale $\left(n^{\circ} 1\right)$ décrit un arc de cercle très prononcé, qui donne à la ventouse une grande profondeur. La charpente rigide étaie une cupule musculaire épaisse. L'ensemble de l'organe fonctionne uniquement comme ventouse.

\section{Morphologie interne' (Pl. I, fig. 4)}

L'examen des coupes montre que l'organisation interne est, à quelques détails près, très semblable à celle que Cerfontaine a décrite pour Diclidophora labracis Cerf. et en revanche assez dissemblable de celle de Diclidophoropsis tissieri.

Appareil digestif. - La bouche (B.) s'ouvre dans un entonnoir buccal, qui mène à un pharynx petit, sphéroïdal. Celui-ci s'ouvre dans un œsophage qui presque immédiatement se divise en deux branches intestinales latérales $(T, d$.). Celles-ci se réunissent dans le plateau adhésif et envoient une digitation dans chaque ventouse. La lumière intestinale est réduite, chaque branche intestinale est profondément découpée et très étalée en largeur. Un épithélium bourré de pigment brun tapisse l'intestin.

Appareil excréteur. - Dans la région céphalique, au niveau de l'œsophage et latéralement, on trouve deux vésicules excrétrices $(V . e$.) bien développées, présentant en coupe une vaste cavité. Chacune de ces vésicules se prolonge par un canal latéral de faible diamètre.

Système nerveux. - Il comporte une bandelette cérébrale $(C$.) bien développée, située au-dessus du pharynx et qui donne naissance à une paire de nerfs antérieurs et à deux paires postérieures, l'une interne, l'autre externe. La paire interne est la plus développée, ses nerfs se rejoignent dans la région du pla- 
teau adhésif, et envoient un filet nerveux dans le pédoncule de chaque ventouse.

Musclas et Parenchyme. - L'un et l'autre sont très peu développés. Les muscles forment une tunique sous-tégumentaire, qui différencie ventralement deux faisceaux de muscles longitudinaux. Les faisceaux musculaires ventraux deviennent plus importants dans la région du plateau adhésif.

\section{Appareil génital (fig. 6)}

Appareil mále. - Les cystes testiculaires (Tes.) sont peu nombreux. Ils sont répartis dans la région postérieure du corps, et médianement, dans l'espace laissé par le tube digestif et les cystes vitellins.

Un canal déférent $(C . d$. $)$ collecte les spermatozoïdes. Ce canal, très étroit dans la région de la glande femelle, se dilate dans la région antérieure en une vésicule séminale allongée. Celle-ci est remplie de spermatozoïdes. Le canal se rétrécit à nouveau au moment où il débouche dans le cirre.

Le cirre est une poche musculeuse, peu développée, ayant un diamètre de $60 \mu$. Les crochets sont peu développés, j'en ai reconstitué huit sur les coupes sériées. Il ne m'a pas été possible de les voir in toto assez clairement pour préciser leur structure.

Appareil femelle. - 1) L'ovaire et les voies génitales femelles : L'ovaire est situé dans la région moyenne du corps proprement dit. Il est constitué par trois lobes qui dessinent ventralement la forme d'un $\mathrm{N}$. Le plus petit lobe est latéral, il contient de très jeunes ovocytes. Le lobe médian contient des ovocytes dont le noyau est au repos, mais dont la taille est incomplète. Enfin le troisième lobe, latéral celui-là, contient des ovocytes ayant atteint leur taille définitive. Ce lobe se poursuit en un oviducte dans lequei s'ouvre un réceptacle séminal $(R . S$.). L'oviducte donne bientòt naissance au canal génito-intestinal ( Ca. g. i.) lequel va s'ouvrir dans une anse intestinale $(T . d$.). A partir du point de confluence avec le canal génito-intestinal, l'oviducte se dirige vers l'arrière, sous l'ovaire, pour décrire ensuite un coude qui l'oriente à nouveau vers la région antérieure au moment où il s'ouvre dans l'ootype après avoir reçu le vitelloducte médian ( $V t \mathrm{~m}$.).

L'ootype (oot.) est moyennement large, allongé, suivant l'axe antéro-postérieur, il reçoit les conduits de nombreuses glandes annexes (Gl. oot). Il se prolonge par un utérus $(U t$.) étroit tout 
d'abord, plus large ensuite, et qui se dilate en une ampoule peu avant de s'ouvrir sous le cirre.

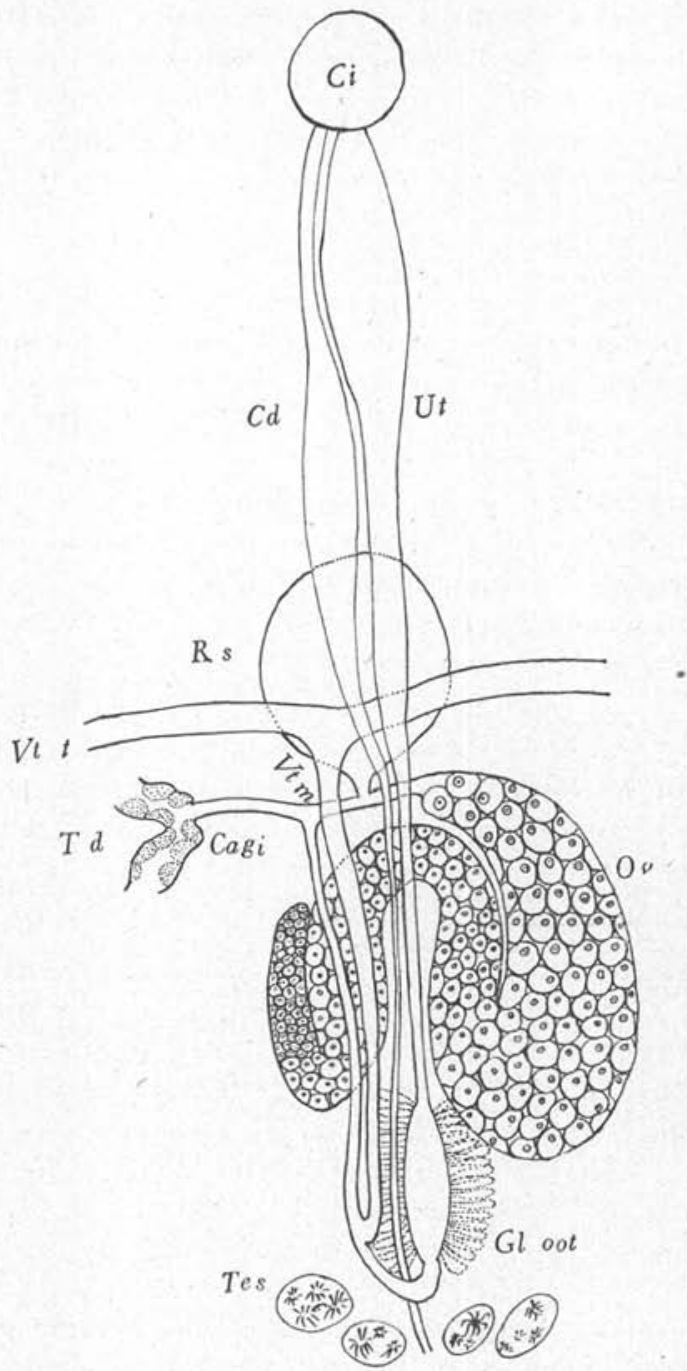

Fit. 6. - Appareil génital de Diclidophora pagelli, vu ventralement.

2) Annexes de l'appareil génital femelle: La glande vitellogène $(V t$.) présente un très grand développement. Les cystes vitellins 
occupent tout le volume du corps, comprimant le tube digestif, et laissent peu de place aux cystes testiculaires. La formation des vitellocytes semḅle continue : on trouve en effet à la fois des nids de jeunes cellules vitellines, et des cellules complètement développées et bourrées de corpuscules coquilliers.

Un peu en avant de l'ovaire et entre le réceptacle séminal qui est dorsal et l'utérus ventral, on trouve les deux branches du vitelloducte transverse $(V t$. $t$.). Celles-ci s'unissent sur la médiane pour se poursuivre en un vitelloducte médian ( $V t . m$.), qui s'ouvre dans l'oviducte, un peu en avant de l'ootype.

Je n'ai observé ni vagins, ni canal vaginal, pas plus que Cerfontaine $(1895 b)$ et Goto (1894), qui ont étudié l'anatomie des autres formes de la famille. Aucune trace ne permet de soupçonner leur présence. Il existe cependant, comme on l'a vu, un réceptacle séminal très développé, bourré de spermatozoïdes. La mise en charge du réceptacle séminal, ses connections, le fonctionnement éventuel du cirre, peu développé, sont des questions que l'examen de cette espèce ne permet pas de résoudre, pas plus que celles étudiées par Goto et Cerfontaine.

\section{Revue systématioue}

Le nom de Diclidophora a été créé par Diesing (1850) pour distinguer des formes confondues jusqu'alors avec d'autres sous le nom d'Octobothrium : D. Iongicollis du Merlan; $D$. palmata de la Molve.

Cerfontaine $(1895 a)$ a montré que ces deux formes doivent être rangées dans le genre Dactylocotyle. Goto (1894) a de nouveau employé le nom de Diclidophora pour désigner quatre espèces :

1) D. smaris vivant sur un Cymothoa de la cavité buccale de Smaris vulgaris.

2) D. elongata, parasite de Pagrus tumifrons.

3) D. sessilis, parasite de la cavité buccale de Chœrops japonicus.

4) D. tetrodonis, parasite de Tetrodon sp.

En 1895 (b) Cerfontaine fait une revue de ces différentes formes. Il décrit une nouvelle espèce $D$. labracis, parasite du Bar. Il détache les formes 3 et 4 de Goto dont il fait les genres Cyclobothrium et Heterobothrium avec $C$. sessilis et $H$. tetrodonis. Il rattache ces genres à la famille des Octocotylidx van Beneden et Hesse, dans laquelle il crée une section des Diclidophorinæ Cerf.

Monticelli (1903) fait des Diclidophorinæ, une sous-famille. 
Linton (1898) décrit Octoplectanum affine dont il fait ensuite (1901) Diclidophora affinis, parasite de Paralichtys dentatus. Plus tard Linton (1904) signale, sans le décrire, un Diclidophora sp. parasite sur Orthopristis chrysopterus L., capturé à Beaufort (Caroline du Nord).

Mac Callum (1917) décrit quatre espèces de Diclidophora:

D. merlangi: branchies de Merluccius bilinearis.

D. prionoti: branchies de Prionotus carolinus.

D. cynoscioni : branchies de Cynoscion regalis.

D. neomenis: branchies de Neomenis analis.

Dollfus (1922) décrit Cyclobothrium charcoti fixé sur Meinertia œstroïdes parasitant lui-même Trachurus trachurus.

Mac Callum (1913) décrit un genre nouveau : Pedocotyle avec Pedocotyle morone, parasite de Morone americana.

Fuhrmann (1929) groupe ces différents genres en une famille des Diclidophoridx, caractérisée essentiellement par le squelette des ventouses. Il est suivi par Sprehn (1933).

Ajoutons enfin que trois espèces peuvent être considérées comme mal définies.

Mesocotyle squillarum Par. et Per. $(1889$ b), parasite de Bopyrus squillarum. Cerfontaine (1898), dans sa revue du genre Dactylocotyle, estime que cette espèce est identique à Diclidophora smaris. L'hôte rend cette opinion assez vraisemblable.

Le genre Choricotyle v. Beneden-Hesse (1863) comporte deux espèces: C. chrysophii, parasite de Chrysophrys aurata et C. (=-Octobothrium) taschenbergii Par. et Perugia, parasite de Sargus rondelettii.

Cette revue terminée, quelle place doit-on attribuer au genre et à l'espèce nouvelle décrits ci-dessus? Remarquons tout d'abord que le caractère fondamental de cette fámille est celui relatif à la structure des ventouses, et notamment à la disposition des pièces de soutien.

Dans tous les cas, où l'étude anatomique des espèces et des genres a été faite, les auteurs, et notamment Cerfontaine (1895), Goto (1894) et Mac Callum (1917) sont d'accord pour n'avoir pas vu de vagins avec certitude. Sprehn (1933), dans sa clé dichotomique, utilise ce fait comme caractère de la famille. Les crochets du cirre sont toujours peu nombreux, de 6 à 8 généralement. Seul Diclidophora (= Octoplectanum) affine Linton possède 15 crochets. Les différents auteurs: Cerfontaine, Goto, Mac Callum, Linton signalent que les crochets sont doubles. 
Ces faits légitiment la création du genre Diclidophoropsis, où les vagins sont indiscutables et où les crochets du cirre sont simples, leur nombre élevé. Par ailleurs, la structure des ventouses ne permet pas de situer ce genre dans une autre famille.

En ce qui concerne Diclidophora pagelli, les caractères tirés du cirre, de l'absence de vagin, des ventouses, permettent une homologation générique facile. L'espèce qui, par l'hôte, en serait le plus voisine, correspond au Choricotyle chrysophrii étudié par Van Beneden et Hesse. Mais l'examen de la planche de ces auteurs, montre que leur espèce à une morphologie différente, l'animal est gracile, la structure des ventouses est bien différente. Au reste, la description sommaire qui en est faite rendra toujours l'homologation de leur espèce difficile.

(A suivre). 